\title{
Influence of Poor Solvents in Radical Polymerizations of Methyl Methacrylate
}

\author{
Tohei Yamamoto, Shigetoshi SeKi, Tadahiro Yamamoto, \\ Masayoshi HIROTA, and Mikiharu KAMACHI* \\ Himeji Institute of Technology, Shosha, Himeji 671-22, Japan \\ * Faculty of Science, Osaka University, Machikaneyama, \\ Toyonaka 560, Japan
}

(Received November 14, 1988)

\begin{abstract}
Radical polymerizations of methyl methacrylate (MMA) were carried out in the presence of poor solvents for poly(methyl methacrylate) (PMMA) at $60^{\circ} \mathrm{C}$. At $40 \mathrm{vol} \%$ or above of $n$-hexane and at $50 \mathrm{vol} \%$ of cyclohexane, the solutions became turbid during polymerization and the polymers precipitated. But the precipitates were not observed in methanol ( $\leqq 50 \mathrm{vol} \%$ ). The kinetic order of the initiator was 0.5 when no precipitate was observed, but it became less than 0.5 when the precipitate was present. Under homogeneous conditions, the values of $(1+x) k_{\mathrm{t}} / k_{\mathrm{p}}{ }^{2}$ were determined from the relations between $1 / P_{n}$ and $R_{p}$, and varied with the viscosity of the system. When a precipitate was observed, $k_{\mathrm{t}} / k_{\mathrm{p}}{ }^{2}$ could not be determined. It is concluded that when a precipitate is observed, the propagating radical grows large enough to precipitate by itself and the termination rate decreases.

KEY WORDS Radical Polymerization / Methyl Methacrylate / Poor Solvent / Inhomogeneous System / Kinetic /
\end{abstract}

Radical polymerizations of vinyl monomers in good solvents have been well studied. ${ }^{1}$ However, radical polymerizations in poor solvents for polymer have not been established well. For example, in the radical polymerization of styrene in poor solvents Barton et al. ${ }^{2}$ reported that $k_{\mathrm{t}}$ (termination rate constnat) was proportional to the reciprocal viscosity of the system, but Cameron et al. ${ }^{3}$ reported that $k_{\mathrm{t}}$ was smaller than that in a good solvent.

Since the above results on styrene polymerization are in disagreement, we investigated radical polymerizations of styrene in poor solvents and got different results ${ }^{4}$ from those as follows: (1) in both methanol $(30,40$, and $50 \mathrm{vol} \%$ ) and isopropanol (40 and $50 \mathrm{vol} \%$ ), turbidity and precipitation were observed, but in cyclohexane and $n$-hexane no turbidity or precipitation was observed; (2) when turbidity and precipitation were observed, the termination rate was markedly enhanced, but when not observed, this rate constant varied with the reverse viscosity of the medium as in good solvents. ${ }^{5}$

In this study, we carried out radical polymerizations of methyl methacrylate (MMA) in methanol, cyclohexane, and $n$-hexane, and found phenomenon contrary to that in styrene polymerization.

\section{EXPERIMENTAL}

MMA was washed with $5 \% \mathrm{NaOH}$ aq solution and water, dried over anhydrous $\mathrm{Na}_{2} \mathrm{SO}_{4}$ and distilled under reduced nitrogen pressure three times. AIBN ( $\alpha, \alpha^{\prime}$-azobisisobutyronitrile) was recrystallized from methanol. Methanol, cyclohexane and $n$-hexane were purified by the methods described in "Organic Solvents."

MMA, AIBN and poor-solvent ( $\geqq 50 \mathrm{vol}^{\circ} \%$ ) were placed in a Pyrex glass tube and the 
mixture was degassed by the freezingpumping-thawing method. Then the tube was sealed off and placed in a thermostat water bath at $60^{\circ} \mathrm{C}$. Poly(methyl methacrylate) (PMMA) was precipitated with cool methanol, filtered and dried under vacuum. The rate of polymerization $\left(R_{p}\right)$ was determined from the weight of polymer. The average degree of polymerization $\left(P_{n}\right)$ was determined from the intrinsic viscosity $([\eta])$ of benzene solution at $30^{\circ} \mathrm{C}$ according to eq 1 . The values of $(1+x) k_{\mathrm{t}} / k_{\mathrm{p}}{ }^{2}$ were determined from the slopes of the relations between $1 / P_{n}$ and $R_{p}$ according to eq 2 .

$$
\begin{aligned}
\log P_{n} & =3.420+1.13 \log [\eta] \\
1 / P_{n} & =(1+x)\left(k_{\mathrm{t}} / k_{\mathrm{p}}{ }^{2}\right)\left(R_{p} /[\mathrm{MMA}]^{2}\right)+C_{\mathrm{m}} \\
& +C_{\mathrm{s}}[\mathrm{S}] /[\mathrm{MMA}]+C_{\mathrm{i}}[\mathrm{AIBN}] /[\mathrm{MMA}](2)
\end{aligned}
$$

\section{RESULTS AND DISCUSSION}

In the radical polymerization of styrene, turbidity and precipitate were observed in methanol $(\geqq 30 \mathrm{vol} \%),{ }^{4}$ while not even turbidity was observed in the polymerization of MMA in methanol. On the other hand, turbidity and precipitation were observed in the polymerizations of MMA in cyclohexane ( 50 $\mathrm{vol}^{\circ} \%$ ) and $n$-hexane ( $\geqq 40 \mathrm{vol} \%$ ), but in the polymerizations of styrene in the above poor solvents, turbidity and precipitation were not observed.

$R_{p}$ determined gravimetrically are listed in Table I and $R_{p}$ dependences on initiator concentration are shown in Fig. 1 (a)-(c). The values of $R_{p}$ for a [methanol] $=10-50 \mathrm{vol} \%$, [cyclohexane] $=10-40 \mathrm{vol}^{\%} \%$ and $[n$-hexane] $=$ $10-40 \mathrm{vol} \%$ in which no turbidity was ob-

\begin{tabular}{|c|c|c|c|c|c|c|c|}
\hline \multirow[b]{2}{*}{ [MMA] } & \multirow{2}{*}{$\begin{array}{l}{[\mathrm{AIBN}]} \\
\times 10^{4}\end{array}$} & \multicolumn{2}{|c|}{ Methanol } & \multicolumn{2}{|c|}{ Cyclohexane } & \multicolumn{2}{|c|}{$n$-Hexane } \\
\hline & & $R_{p} \times 10^{5}$ & $(1+x) k_{t}$ & $R_{p} \times 10^{5}$ & $(1+x) k_{\mathrm{t}}$ & $R_{p} \times 10^{5}$ & $(1+x) k_{\mathrm{t}}$ \\
\hline $\mathrm{vol}^{\circ} \%$ & $\mathrm{~mol} \mathrm{l}^{-1}$ & $\mathrm{moll}^{-1} \mathrm{~s}^{-1}$ & $k_{\mathrm{p}}^{2}$ & $\mathrm{~mol} \mathrm{l}^{-1} \mathrm{~s}^{-1}$ & $k_{\mathrm{p}}^{2}$ & $\mathrm{~mol} \mathrm{1}^{-1} \mathrm{~s}^{-1}$ & $k_{\mathrm{p}}^{2}$ \\
\hline \multirow{4}{*}{90} & 4.92 & 7.07 & & 6.56 & & 6.46 & \\
\hline & 2.46 & 4.95 & 53 & 4.78 & 64 & 4.54 & 61 \\
\hline & 1.22 & 3.58 & & 3.30 & & 3.25 & \\
\hline & 0.61 & 2.44 & & 2.36 & & 2.32 & \\
\hline \multirow{4}{*}{80} & 4.92 & 6.60 & & 5.75 & & 5.31 & \\
\hline & 2.46 & 4.54 & 54 & 4.08 & 63 & 3.58 & 70 \\
\hline & 1.22 & 3.26 & & 2.88 & & 2.64 & \\
\hline & 0.61 & 2.31 & & 2.01 & & 1.86 & \\
\hline \multirow{4}{*}{70} & 4.92 & 6.00 & & 4.91 & & 4.10 & \\
\hline & 2.46 & 4.13 & 55 & 3.46 & 68 & 2.83 & 87 \\
\hline & 1.22 & 2.91 & & 2.50 & & 2.02 & \\
\hline & 0.61 & 2.07 & & 1.69 & & 1.45 & \\
\hline \multirow{4}{*}{60} & 4.92 & 5.14 & & 4.08 & & 3.62 & \\
\hline & 2.46 & 3.51 & 57 & 2.90 & & 2.82 & \\
\hline & 1.22 & 2.59 & & 2.09 & & 2.14 & \\
\hline & 0.61 & 1.81 & & 1.40 & & 1.73 & \\
\hline \multirow{4}{*}{50} & 4.92 & 4.42 & & 3.33 & & 2.56 & \\
\hline & 2.46 & 3.03 & 54 & 2.72 & & 2.03 & \\
\hline & 1.22 & 2.18 & & 2.14 & & 1.33 & \\
\hline & 0.61 & 1.51 & & 1.56 & & 1.04 & \\
\hline
\end{tabular}
served were proportional to the square root

Table I. Results of radical polymerizations of MMA in poor-solvents at $60^{\circ} \mathrm{C}$ 


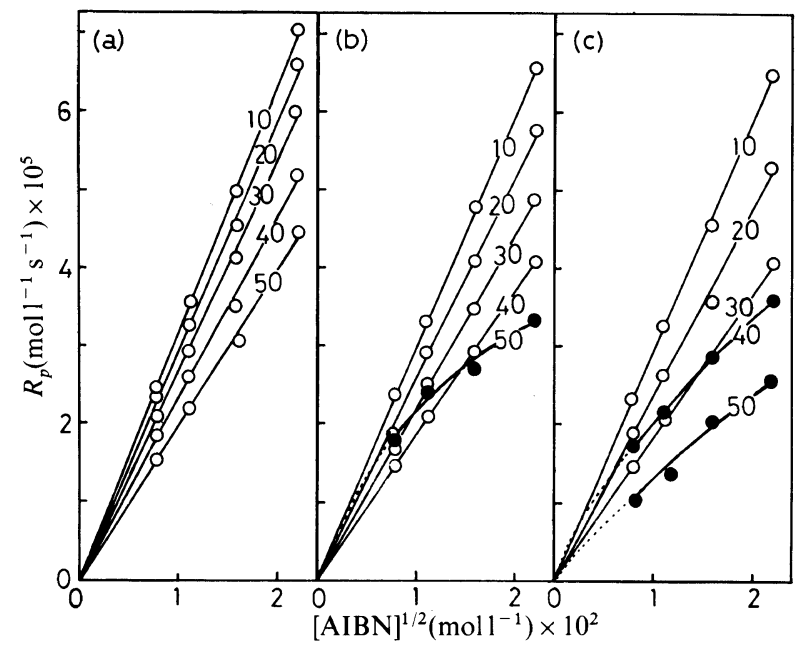

Figure 1. Dependence of $R_{p}$ on AIBN concentration in methanol: (a), cyclohexane; (b), $n$-hexane, (c), at $60 \mathrm{C}$. Numbers and black circles in the figures indicate $\mathrm{vol}^{\circ} \%$ of poor-solvent and precipitation, respectively.

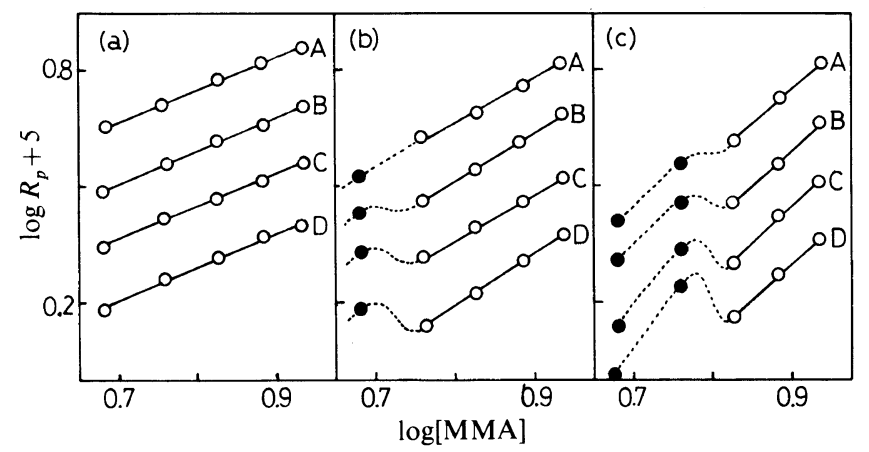

Figure 2. Dependence of $R_{p}$ on MMA concentration in methanol; (a), cyclohexane; (b), $n$-hexane; (c), at $60^{\circ} \mathrm{C}$. Black circles indicate precipitation. $[\mathrm{AIBN}]\left(\mathrm{moll}^{-1}\right) \times 10^{4}: \mathrm{A}, 4.92 ; \mathrm{B}, 2.46 ; \mathrm{C}, 1.22 ; \mathrm{D}, 0.61$.

of [AIBN] as shown in Figure 1. However, the plots of $R_{p}$ in the cases of [cyclohexane] $=50$ $\mathrm{vol}^{\%} \%$ and $[n$-hexane $]=40$ and $50 \mathrm{vol} \%$ against the square root of [AIBN] deviated from the proportional relation, especially at low [AIBN]. Although the deviation is similar to that caused by the primary radical termination, it is not caused by primary radical termination. ${ }^{7}$ Because in these cases, $R_{p}$ increased at low [AIBN] as shown in Figure 1, while $R_{p}$ should decrease at high [AIBN] in the case of primary termination.

The dependence of $R_{p}$ in methanol, cyclo- hexane and $n$-hexane on [MMA] is shown in Figure 2(a)-(c). The plots of $\log R_{p}$ against $\log [\mathrm{MMA}]$ in methanol showed linear relationships as in Figure 2(a), but the plots in cyclohexane and $n$-hexane deviated markedly with MMA concentration of precipitate observed as shown in Figure 2(b), (c). The deviation was remarkable at low [AIBN].

From these results, we consider the following: As a propagating radical becomes large enough to coil and poor solvent can separate the polymer radical and solution, the end radical should be occluded in the coil. So the 


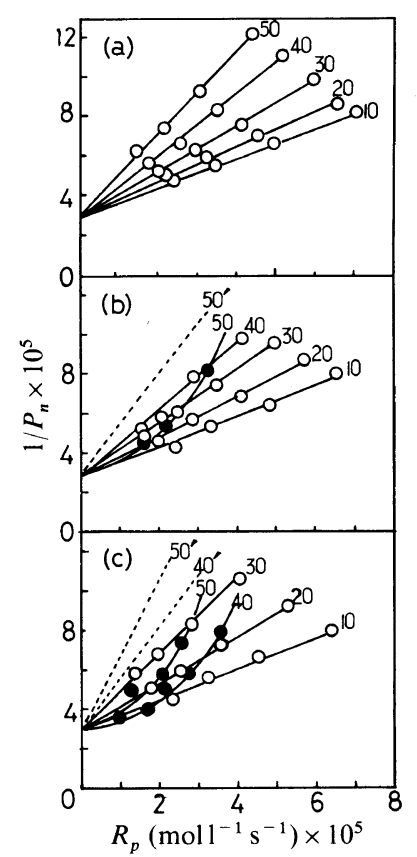

Figure 3. Relations between $1 / P_{n}$ and $R_{p}$ in methanol; (a), cyclohexane; (b), $n$-hexane; (c), at $60^{\circ} \mathrm{C}$. Numbers and black circles in the figures indicate vol $\%$ of poorsolvent and precipitation, respectively. The broken lines indicate the relations supposed if the system was homogeneous. The same intercept as solid line was taken because of small chain transfer constant to cyclohexane or $n$-hexane and the slope was derived on the basis of $(1+x) k_{\mathrm{t}} / k_{\mathrm{p}}^{2}$ determined from the relation in Figure 4 .

termination reaction is suppressed, but the propagation reaction should not be affected. As a result, $R_{p}$ is enhanced when a precipitate is observed.

In order to examine the influence of poor solvents on the termination step, $1 / P_{n}$ was plotted against $R_{p}$ in Figure 3(a)-(c). As shown in Figure 3, linear relationships were obtained in methanol $(10-50 \mathrm{vol} \%$ ), cyclohexane $\left(10-40 \mathrm{vol}^{\mathrm{o}} \%\right)$, and $n$-hexane $(10-30$ $\mathrm{vol}^{\mathrm{o}} \%$ ). It suggests that under homogeneous conditions, polymerization proceeds by the ordinal mechanism of radical polymerization. The values of $(1+x) k_{\mathrm{t}} / k_{\mathrm{p}}{ }^{2}$ were determined from the slopes and are listed in Table I. The plots of the values against the reciprocal of the viscosity of the system were almost on the line

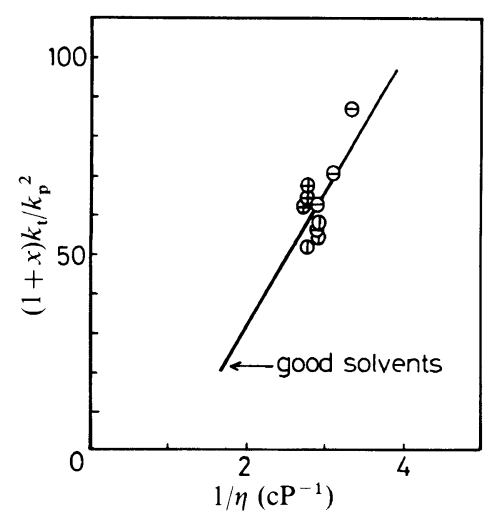

Figure 4. Plots of $(1+x) k_{\mathrm{l}} / k_{\mathrm{p}}{ }^{2}$ against $1 / \eta$. (1), methanol; $\oplus$, cyclohexane; $\ominus, n$-hexane.

obtained for the polymerizations in good solvents (Figure 4). This also indicates that polymerizations in these systems proceed by the same manner as in good solvents and poor solvents do not affect the termination process unless polymer precipitates.

On the other hand, when a precipitate was observed $\left([\right.$ cyclohexane $]=50 \quad \mathrm{vol}_{\%}^{\circ}$ and $[n$ hexane] $=40$ and $50 \mathrm{vol} \%$ ), the plots deviated far below the relations (broken lines in Figure 3) supposed if the systems were homogeneous, and showed curves. Therefore the values of $(1+x) k_{\mathrm{t}} / k_{\mathrm{p}}^{2}$ in these cases could not be determined, but the values estimated from the tangent of the curves were smaller than those from the broken lines.

We consider from the smaller $(1+x) k_{\mathbf{t}} / k_{\mathrm{p}}{ }^{2}$ and larger $R_{p}$ at lower AIBN concentration that the propagating PMMA radical becomes large enough to precipitate by itself, the radical is occluded in the coil and termination reaction is prevented.

Contrary to the poly MMA radical, the propagating polystyryl radicals were not large enough to precipitate by themselves, radicals clustered in poor solvent and termination reaction was enhanced. After termination, the polymers gathered and precipitated.

The main issue is precipitation. Cameron et $a l .{ }^{3}$ also studied the radical polymerizations of MMA in poor solvents but did not mentioned 
the importance of precipitation. It is concluded that poor solvents are not different from good solvents until polymer precipitates.

\section{REFERENCES}

1. G. M. Burnett, W. S. Dailey, and J. M. Person, Trans. Faraday Soc., 61, 1216 (1965); G. H. Olive, and S. Olive, Makromol. Chem., 96, 221 (1966); C. H. Bamford and S. Olive, Makromol. Chem., 96, 221 (1966); C. H. Bamford and S. Brumby, ibid., 105, 122 (1967); M. Kamachi, D. J. Liaw, and S. Nozakura, Polym. J., 11, 921 (1979); T. Yamamoto, T. Yamamoto, Ly Phat, and M. Hirota, Nippon Kagaku
Kaishi, 569 (1981).

2. J. Barton, I. Kapek, V. Juranicova, and M. Mlynarova, Makromol. Chem., 181, 1289 (1980).

3. G. G. Cameron and J. Cameron, Polymer, 14, 107 (1973).

4. T. Yamamoto, S. Seki, M. Kamachi, T. Yamamoto, and M. Hirota, Nippon Kagaku Kaishi, 1585 (1987); T. Yamamoto, S. Seki, M. Kamachi, and T. Yamamoto, ibid., in press (1989).

5. T. Yamamoto, T. Yamamoto, T. Yamamoto, and M. Hirota, Nippon Kagaku Kaishi, 618 (1980).

6. J. A. Riddik and W. A. Bunger, "Organic Solvents," 3rd ed, Wiely-Interscience, New York, N.Y., 1970.

7. G. S. Misra, A. Hafeez, and K. S. Sharma, Makromol. Chem., 51, 123 (1962). 